Revue internationale P.M.E.

Économie et gestion de la petite et moyenne entreprise

\title{
Étude qualitative et comparative sur la portée des relations entre la recherche et la communauté d'entreprises dans les pôles technologiques
}

\author{
Isidre March-Chordá et Rosa M. Yague-Perales
}

Volume 11, numéro 1, 1998

URI : https://id.erudit.org/iderudit/1009037ar

DOI : https://doi.org/10.7202/1009037ar

Aller au sommaire du numéro

Éditeur(s)

Presses de l’Université du Québec

ISSN

0776-5436 (imprimé)

1918-9699 (numérique)

Découvrir la revue

Citer cette note

March-Chordá, I. \& Yague-Perales, R. M. (1998). Étude qualitative et comparative sur la portée des relations entre la recherche et la communauté d'entreprises dans les pôles technologiques. Revue internationale P.M.E., 11(1), 81-100. https://doi.org/10.7202/1009037ar
Résumé de l'article

Tant par le degré de participation des agents scientifico-technologiques du milieu local que par les relations avec les entreprises implantées sur les pôles technologiques, la promotion et le développement d'espaces technopolitains sont très diversifiés selon le site et la région.

Compte tenu de cette diversité, nous avons entrepris une étude basée sur l'estimation qualitative d'une série de variables concernant le comportement des universités et centres de recherche dans 13 pôles technologiques. Cela nous a permis de conclure que l'intensité scientifique et technique à l'intérieur du pôle et la relation réelle entre les agents de recherche et les entreprises locataires sont les conditions les plus valables pour avancer vers la participation optimale de la communauté scientifico-technologique locale dans tous les domaines de la vie d'un pôle d'entreprises de haute technologie. 


\section{Étude qualitative et comparative sur la portée des relations entre la recherche et la communauté d'entreprises dans les pôles technologiques}

Isidre MARCH-CHORDÁ

Rosa M. YAGUE-PERALES

Université de Valence, Espagne

MOTS CLÉS

\section{Pôles technologiques - Innovation - Technologie Universités - Centres de recherche}

\section{RÉSUMÉ}

Tant par le degré de participation des agents scientifico-technologiques du milieu local que par les relations avec les entreprises implantées sur les pôles technologiques, la promotion et le développement d'espaces technopolitains sont très diversifiés selon le site et la région.

\section{LES AUTEURS}

Isidre March-Chordá a obtenu son doctorat en économie en mai 1994. Auparavant, en 1991, il avait obtenu une M.Sc. en technologie et management de l'innovation au SPRU de l'Université de Sussex, Royaume-Uni. Actuellement, Isidre March-Chordá est professeur au Département de management d'entreprises de l'Université de Valencia, en Espagne, où il travaille dans divers projets de recherche concernant la gestion de la technologie et la création d'entreprises innovatrices. Adresse: Campoamor 101, 36 - 46022 Valencia, Espagne. Courriel: Isidre.March@uv.es

Rosa M. Yague-Perales est professeure titulaire au Département d'économie appliquée à l'Université de Valence. Ph.D. depuis 1996, elle est spécialiste de l'application de techniques statistiques à l'économie et au management d'entreprises. Elle est auteure de plusieurs articles publiés dans des revues internationales. 
Compte tenu de cette diversité, nous avons entrepris une étude basée sur l'estimation qualitative d'une série de variables concernant le comportement des universités et centres de recherche dans 13 pôles technologiques. Cela nous a permis de conclure que l'intensité scientifique et technique à l'intérieur du pôle et la relation réelle entre les agents de recherche et les entreprises locataires sont les conditions les plus valables pour avancer vers la participation optimale de la communauté scientifico-technologique locale dans tous les domaines de la vie d'un pôle d'entreprises de haute technologie.

\begin{abstract}
When analysing the technopolitan phenomenon it is remarkable how both the degree of commitment by the local scientific and technological sources in the development of the technopolitan space and the extent of sinergies between the scientific community and the tenant firms, significantly differ depending on the region and site.

Keeping this diversity in mind, this paper tries to provide an assessment on the role played and behavior exhibited by the HEls and Research Centres in the technological poles, as well as their exchange with the tenant firms. From the empirical analysis on 13 technological poles in France, United Kingdom and Belgium, we derive the scientific and technological intensity within the park, together with the actual links between the entrepreneurial and scientific community, are the most valuable requirements to attain an optimal involvement by the research community in the parks.
\end{abstract}

\title{
RESUMEN
}

Tanto el grado de participación de los agentes cientifico-técnicos del entorno local en la creación y desarrollo de los espacios tecnopolitanos, como su grado de relación con las empresas implantadas en dichas áreas, varían enormemente según las zonas y regiones.

Tras resaltar esta diversidad, el presente estudio ofrece una estimación cualitativa sobre una serie de variables que reflejan el peso y comportamiento de la comunidad universitaria e investigadora en los parques tecnológicos. Este análisis nos propone la intensidad científico-investigadora en el interior del parque y el alcance real de las relaciones entre las fuentes cientifico-técnicas y las empresasparque, como las condiciones más valiosas para lograr una participación óptima de la comunidad científico-tecnológica local en el desarrollo de los parques tecnológicos.

\section{Introduction}

La présente analyse a pour but de mesurer diverses expériences dans les pôles technologiques (PT) à partir d'études directes de différents cas réels.

Depuis le début des années 1980, ce qu'on appelle les pôles technologiques, pôles de recherche, technopoles, soit autant de termes englobés ici sous l'acception 
générique de pôles technologiques (PT), prolifèrent et acquièrent une importance croissante dans le cadre des politiques industrielles des pays industrialisés, surtout aux États-Unis, en Europe et au Japon, où ces expériences sont considérées comme des moteurs pour les activités et les secteurs de technologies de pointe. En raison de la diversité des formes et des objectifs que ces espaces présentent selon les régions et les pays, il ne s'avère toutefois pas facile de trouver une définition des PT qui puisse être internationalement reconnue. L'observation de ces expériences, réparties dans le monde entier, nous conduit à les voir comme des espaces délimités territorialement et destinés à accueillir des activités de technologie de pointe présentant un haut degré d'innovation par rapport aux secteurs traditionnels prédominants dans le cadre local ou régional.

Jusqu'à présent, les PT ont été principalement promus par les autorités locales, régionales et les universités. Cette promotion des autorités publiques, dans la plupart des PT, découle de leur confiance dans la capacité des PT à avoir des effets bénéfiques sur les économies locales et régionales qui les entourent, jouant ainsi le rôle d'instrument de politique territoriale d'une région déterminée.

Actuellement, un bon nombre de régions européennes étudient de manière plus ou moins approfondie l'utilité et l'aptitude des pôles technologiques et des technopôles à servir de première étape pour le lancement de projets de haute technologie. En même temps, dans d'autres régions, les PT, dont les débuts remontent à plus de 10 ans, sont largement connus et s'approchent déjà d'une certaine maturité qui permet aux spécialistes de tirer des conclusions et des leçons de leurs évolutions, de leur impact et de leurs perspectives.

\section{But de l'étude}

Avec cette étude, laquelle s'appuie sur la documentation existante ${ }^{1}$, mais surtout sur nos propres analyses empiriques, nous espérons éclairer davantage la situation, les caractéristiques et, enfin, la relation entre les entreprises innovatrices dans le cadre des PT. En même temps, nous poursuivons d'autres objectifs que nous pouvons formuler dans les termes suivants :

- analyser certains dilemmes qui subsistent au sujet des PT;

1. Cet article, basé sur l'expérience observée dans une série de pôles technologiques et les technopôles, s'inscrit dans la ligne suivie ces dernières années par d'autres auteurs. Parmi les plus importants, nous pouvons mentionner Quintas et al. (1991), Bruhat (1991), Bruhat, Escorsa et al. (1995) et Castells et Hall (1994). Tout comme leurs études, la nôtre établit une comparaison entre diverses expériences faisant partie d'un même phénomène, mais nous y apportons peut-être un aspect plus qualitatif élaboré à travers le plan de travail suivi pendant les séjours dans les zones étudiées. 
- fournir une méthodologie d'évaluation qui puisse servir de guide afin d'estimer la situation de quelques domaines clés dans les parcs existants ;

- explorer les possibilités offertes par les expériences en cours d'étude et de planification aux entreprises et agents de recherche intéressés par ces espaces innovateurs.

Les étapes à suivre seront d'abord une exploration des raisons des rapports entre PME et pôles technologiques, l'intérêt des entreprises pour ces espaces et les mécanismes de financement des firmes technopolitaines. Puis, nous aborderons le noyau de notre analyse, axé sur une série de comparaisons et de corrélations en rapport avec un fait d'importance particulière : d'une part, le rôle des universités et des centres de recherche et, d'autre part, l'interprétation des liens et des synergies recherche-entreprise dans les pôles technologiques grâce à des méthodes statistiques.

Nous nous sommes proposé aussi de mettre en relief le véritable rôle joué par la communauté scientifique dans le développement des pôles technologiques et des entreprises qui s'y installent.

Les résultats sont l'objet d'une interprétation qualitative détaillée, basée sur notre étude empirique et composée de 13 expériences réelles de PT du RoyaumeUni, de la France et de la Belgique.

\section{Les rapports entre PME et pôles technologiques}

\subsection{Les PME et les pôles technologiques}

Sans doute, la plupart des agents locataires aux pôles technologiques sont des PME.

Une telle majorité épouse les désirs des agents dirigeants et des promoteurs de ces espaces qui, d'habitude, fondent leurs préférences pour ce type d'entreprises sur les faits suivants :

- les PME bénéficient des avantages «de comportement » liés à un degré élevé de versatilité et d'adaptabilité aux changements expérimentés par leur environnement ;

- les PME les plus dynamiques et les plus innovatrices sont portées à interagir avec le milieu économique local ;

- on présume que les PME situées sur un pôle technologique sont capables d'avoir des effets notables plus vastes et plus intenses sur le tissu productif local, du fait qu'elles deviennent des canaux de diffuseurs des avantages rattachés à l'adoption des nouvelles technologies. Cette capacité s'explique par de grandes similitudes entre les PME des pôles et les entreprises qui composent le tissu industriel de la plupart des 
régions, par rapport à des variables clés comme leur dimension, leur origine et leurs marchés ;

- il est plus probable que la présence massive de PME confère aux PT un degré d'indépendance, d'autonomie et de diversification plus important, ce qui permet d'optimiser leur impact sur le milieu local ;

- en principe, on estime plus facile de créer des synergies et d'établir des relations de coopération et d'échange dans une agglomération formée de PME.

Après avoir exposé quelques motifs qui incitent les promoteurs des PT et ou autres formes de projet du genre à accueillir des PME innovatrices, nous exposons tout de suite les points forts et les défaillances reliés à l'admission des grandes entreprises dans un espace de PT.

Mentionnons quelques aspects les plus avantageux :

- l'arrivée de quelques grandes corporations favorise une rapide occupation des terrains et des immeubles disponibles dans les pôles. Cela réduit la période nécessaire pour amortir les investissements effectués pour l'aménagement des espaces technopolitains ;

- d'habitude, l'implantation des grandes entreprises sur un PT nécessite de forts investissements qui favorisent une rapide création d'un nombre considérable d'emplois, tout en bénéficiant de la richesse globale de l'économie locale ;

- l'entrée de quelques grandes et prestigieuses multinationales dans un PT augmente sa crédibilité, améliore son image autant sur le plan international que national.

Ces avantages n'assurent pas d'effets positifs nets en ce qui concerne le développement équilibré et de longue durée du milieu local, puisqu'ils peuvent être annulés par la présence d'autres effets nuisibles. Parmi les aspects problématiques les plus communs, résultant d'une présence dominante de grandes entreprises sur les espaces PT, on peut repérer:

- une perte d'autonomie sur la trajectoire du pôle technologique, laquelle reste fortement liée aux projets et aux préférences exprimées par ces entreprises dominantes;

- un effet d' « introversion » qui se traduit par un plus faible échange avec le milieu local, avec pour résultat des comportements isolationnistes ;

- ces entreprises tendent à recruter le personnel le plus qualifié du marché local, ce qui pourrait laisser les PME locales dépourvues des meilleures compétences ; 
- en général, les grandes sociétés considèrent les pôles technologiques comme de simples outils pour déployer leurs propres stratégies de décentralisation ;

- les unités de grande taille tendent à monopoliser les relations avec les centres scientifico-techniques régionaux ;

- enfin, une présence massive de grandes entreprises sur un espace technopolitain est considérée comme peu favorable à l'esprit d'entrepreneuriat du milieu local.

\subsection{Mécanismes de financement des entreprises PT}

De bonnes sources de financement conventionnelles ainsi que l'accès aux fonds de capitaux participatifs, tels que les formules de capital-risque et de «capital de démarrage » (seed capital), sont des conditions habituellement qualifiées d'essentielles pour le démarrage et les premières phases de vie des PME innovatrices.

Hormis leur but lucratif, la plupart des sociétés de capital-risque se constituent dans le but de favoriser la naissance et de stimuler le développement de nouvelles entreprises douées d'une certaine puissance innovatrice.

Généralement, l'implantation de ces nouvelles entreprises est prioritaire pour les pôles technologiques. Cependant, et après quelques années d'expérience dans les marchés britannique, américain et français, les diverses formules de capitalrisque existantes ne sont jamais parvenues à atteindre un niveau de réussite et de diffusion significatifs.

Cette incidence plutôt faible des nouveaux mécanismes de financement sur les marchés des entreprises naissantes et innovatrices explique la survivance de conditions financières peu favorables à la création des PME douées d'un potentiel innovateur élevé, justement celles que l'on souhaite voir s'installer sur un PT.

Seules quelques zones des États-Unis, telles que la Bay Area de San Francisco, de Silicon Valley et la région de Boston, ont été capables de développer un marché de capital-risque très actif et dynamique, grâce à un généreux financement de nouveaux projets d'entreprises axées sur des secteurs de pointe.

Par contre, en Europe, les fournisseurs de capital-risque affichent des stratégies d'investissement très prudentes et très sélectives : ils ne donnent leur soutien qu'aux entreprises jouissant de perspectives de développement exceptionnelles et qui ont des équipes de gestion expérimentées et compétentes. 


\subsection{Attrait des PT pour les entreprises à technologie de pointe}

On a pu constater que les entreprises les plus souvent implantées dans les secteurs de PT présentent un degré de perfectionnement technologique moyennement élevé, combiné à une certaine spécialisation dans des activités de R-D, dessin et développement de prototypes.

En principe, l'intérêt de ce type d'entreprises pour les milieux innovateurs comme les PT peut s'expliquer par diverses raisons. À notre avis, les plus significatives sont exposées ci-dessous ${ }^{2}$ :

- le composant immobilier : l'accès à des terrains, immeubles et locaux offrant des conditions avantageuses ;

- la localisation de l'espace PT dans un milieu urbain bien équipé de services et bien pourvu en voies de communication ;

- le facteur agglomération : l'espace PT compris comme une agglomération d'entreprises où il est facile d'atteindre des économies croissantes d'échelle ;

- la proximité des sources de recherche scientifique-technologique, afin d'accéder plus facilement aux équipements technologiques et aux services d'assistance technologique fournis par les chercheurs et par le personnel technique qui travaillent dans ces centres ;

- le flux potentiel d'échange réciproque d'information à l'intérieur du PT entre les locataires et entre les entreprises et le milieu institutionnel, économique et social de la zone ;

- la connaissance et le contact des éventuels partenaires en vue d'entamer des relations de collaboration;

- l'effet de l'image : la seule appartenance à un espace PT reconnu et prestigieux permet aux entreprises locataires d'être qualifiées comme des entreprises PT ou technopolitaines ; cela équivaut à un certain contrôle sur les technologies pointues et l'offre d' « outputs avancés ». De cette façon, les PT confèrent aux entreprises situées sur leurs espaces une crédibilité et un prestige accrus ;

- les meilleures possibilités d'ouverture à l'échange de personnel de la part des entreprises, des centres de recherche et des laboratoires de R-D publics et privés. Cet échange permet éventuellement d'augmenter la qualification du personnel technique des entreprises.

2. D'après notre étude empirique et aussi selon les travaux de Gamella (1988) et Quintas et al. (1991). 


\section{3. Étude empirique}

Nous résumons ici les faits saillants concernant le comportement récent des entreprises locataires dans des PT, à partir d'une série d'études publiées récemment ${ }^{3}$ et, en particulier, sur nos propres travaux de terrain entrepris au Royaume-Uni (1991) et en France (1992) sur les PT ${ }^{4}$.

\subsection{Intérêt des PT pour la communauté scientifique et de recherche}

Les universités et les centres publics de recherche sont les promoteurs habituels des pôles technologiques. En effet, beaucoup de PT sont installés sur les campus universitaires ou à proximité.

Parmi les motivations et les faits dynamisants les plus communs qui attirent la participation active des membres de corps professoraux et de chercheurs dans la promotion et même dans la gestion d'un PT, quelques-uns méritent d'être relevés.

- L'occasion de tirer des revenus après la signature de contrats et d'accords de collaboration avec les entreprises placées sur le PT. Cela offre la possibilité d'exploiter d'une manière plus profitable les terrains, les immeubles, les laboratoires et l'équipement de recherche, ainsi que d'autres ressources des universités et des centres de recherche.

- Le PT, en tant que milieu d'activité productive, est susceptible d'encourager la vocation d'entrepreneur et d'inciter le personnel de recherche à exploiter ses talents d'entrepreneur.

- Le PT peut capter et utiliser des idées fournies par les entreprises pour enrichir le savoir-faire des départements de recherche qui participent à la promotion de ces espaces.

3. Les études de Quintas et al. (1988 et 1991), Gamella (1988), Saxenian (1988), Giunta (1993), Bruhat (1991), Castells et Hall (1994). Nous avons également consulté des études qui établissent un rapport entre les variables territoire et innovation, études comme celles de Perrin, Quevit, Decoster, Allaman, Gaffard, Maillat (toutes mentionnées dans la bibliographie); certains d'entre eux appartenant à des groupes d'analystes connus tel le GREMI.

4. La méthodologie employée dans l'étude empirique a consisté dans la visite et l'entretien personnel avec les principaux promoteurs, directeurs et gestionnaires de pôles technologiques et technopôles énumérés plus loin. Nous avons également rencontré les dirigeants de quelques entreprises situées sur ces sites et les organismes responsables de la politique industrielle et de la promotion de l'innovation sur le plan régional. 
- L'interaction avec une communauté d'entreprises potentiellement innovatrices comme celles des PT permet d'élargir les perspectives et d'enrichir l'avis des chercheurs sur la réalité qui entoure le monde des entreprises.

- Les PT, en tant que portes d'accès à l'extérieur des institutions et par les moyens dont ils disposent pour diffuser leurs compétences, peuvent attirer de nouveaux clients intéressés aux services et aux projets des départements universitaires de recherche.

\subsection{Relations entre les entreprises et les universités ou centres de recherche}

Le rôle joué par la communauté universitaire et les centres de recherche dans la dynamique des PT differe sensiblement selon les cas. Il dépend de plusieurs facteurs dont les plus significatifs sont les suivants : le prestige attaché au réseau scientifique et technique, la stratégie de rapprochement vers l'université suivie par la direction du PT, les synergies des départements et des centres de recherche avec l'économie régionale, l'apport public à l'activité de recherche de ces centres, la politique de formation du personnel universitaire et des chercheurs.

L'implication directe d'un centre de formation supérieure dans la mise sur pied et la gestion d'un PT peut prendre des formes différentes. En voici quelquesunes exposées selon des modes opérationnels :

- fournir des terrains pour que le pôle technologique puisse être implanté sur le campus universitaire ;

- favoriser l'accès des entreprises du pôle à l'équipement matériel des départements universitaires ;

- accéder aux connaissances et aux autres compétences intangibles détenues par les équipes de recherche ;

- stimuler l'essaimage des entreprises à partir de la recherche.

\subsection{Méthodologie}

Les pôles qui ont fait l'objet de l'étude empirique sont décrits au tableau 1.

Avec cet échantillon d'initiatives, la diversité est assurée, car on peut trouver des pôles expérimentés dont la fondation remonte à 10 ou 12 ans et d'autres très récents, tels ceux d'Angers et de Chambéry.

À notre avis, l'étude empirique menée sur ces pôles technologiques est suffisamment détaillée et approfondie pour élaborer un tableau avec des valorisations qualitatives sur le degré d'implication des universités et des centres de recherche 


\section{TABLEAu $1^{*}$ \\ Description des pôles technologiques à l'étude}

\section{ROYAUME-UNI}

- Surrey Research Park : situé près de Londres, c'est le plus vaste des pôles britanniques étudiés. Il est présenté comme un parc de recherche consacré exclusivement aux activités de R-D.

- Aston Science Park : situé au centre de la ville de Birmingham, il regroupe surtout des entreprises utilisatrices de technologies avancées. Il offre une large gamme de services à ses locataires, parmi lesquels un fonds de capital-risque.

- Sheffield Science Park : situé dans la ville, il est plus petit que les précédents et est formé de deux bâtiments modulaires qui accueillent une trentaine de sociétés de services avancés.

- Warwick Science Park : situé près la ville de Coventry, à côté de Birmingham, il est de taille similaire à l'Aston Science Park. Son principal objectif est le transfert de technologie entre l'Université de Warwick et l'industrie locale.

\section{FRANCE}

- Lyon Technopolis : articulé autour de quatre pôles technologiques, il cherche à restructurer le tissu industriel de la métropole lyonnaise et à stimuler l'échange RechercheEntreprise.

- Montpellier Technopole : créé sous l'initiative du district de Montpellier, il regroupe la totalité des zones d'activité et des aires industrielles situées dans la ville et en périphérie, parmi lesquelles seules quelques-unes accueillent un nombre significatif d'entreprises avancées.

- Montpellier Technopole Régionale : une initiative de la région Languedoc-Roussillon ; il cherche à susciter l'apparition et la consolidation de pôles scientifico-technologiques dans la région. Étant à l'état naissant en 1993, ses objectifs n'étaient pas entièrement définis.

- Angers Technopole : de création récente, il vise à promouvoir la création d'entreprises innovatrices, renforcer la R-D dans le milieu local et susciter un climat de collaboration entre les agents économiques locaux.

- Atlanpole Nantes : il se donne comme objectif de favoriser la rencontre entre les sources de recherche locales et le tissu industriel. Il se distingue par son exposé endogène basé sur la promotion d'essaimage à partir de la recherche.

- Savoie Technolac : situé à Chambéry, dans la région Rhône-Alpes, il est toujours à l'état naissant. Ses objectifs sont larges et non restreints quant à ses capacités locatives. Il abrite les écoles scientifico-techniques de l'université locale.

- Rennes Atalante : de nature multipolaire, on peut y remarquer la création de « spin-offs » de recherche. Il est plutôt spécialisé dans le secteur de la télématique.

- Cité Scientifique Île-de-France Sud : ce pôle regroupe quelque 50 municipalités et s'étend sur le territoire ayant la plus grande densité scientifique en France. Sa faible représentation face aux collectivités locales le relègue au rôle d'organe consultatif.

\section{BELGIQUE}

- Louvain-la-Neuve : l'objectif du PT de cette ville nouvelle et de son instigateur, l'Université de Louvain-la-Neuve, est de créer un nouveau tissu urbain intégré harmonieusement avec l'université.

* Les données qui apparaissent dans ce tableau renvoient aux années 1993 et 1994. 
dans la vie des PT et les rapports entre entreprise et recherche sur chacun des 13 cas réels de pôles technologiques visités.

C'est à partir de l'information présentée dans le tableau $2 a$ que nous avons procédé à une analyse statistique composée d' une étude qualitative et comparative des 13 PT visités et à une analyse factorielle qui nous a conduits à l'obtention de trois facteurs ou indicateurs synthétiques (voir le tableau $2 a$ ).

Cette analyse factorielle est prise dans le sens de la version des composants principaux et sous un critère de détermination des facteurs VARIMAX. Ce critère nous permet d'expliquer un maximum d'information avec le moins de variables possible, en fixant un minimum de variabilité qui doit être expliquée par ces facteurs. Nous avons choisi deux facteurs qui expliquent $82,4 \%$ de la variance totale.

Ensuite, sur la base de cette analyse factorielle, le degré d'accomplissement de ces trois facteurs à chacun des pôles étudiés est contrasté par le tableau $2 b$. Ce tableau représente la matrice des coefficients de ponctuation des facteurs (Factor Score Coefficient Matrix), obtenue à partir de la rotation de la matrice des facteurs (Rotated Factor Matrix). Finalement, le même tableau $2 b$ nous apporte une valeur globale qui indique la position de chaque PT par rapport à l'ensemble de ces trois facteurs.

\subsection{Résultats empiriques}

Le tableau $2 a$ illustre notre vision qualitative des liens réels tissés entre les entreprises du PT et la communauté scientifique implantée dans le pôle ou dans sa périphérie, pour chacun des PT étudiés.

Les cinq variables soumises à l'étude ont été choisies a priori pour leur capacité explicative du rôle joué par les institutions de recherche sur les environnements technopolitains.

\section{Variable 1: Centre de recherche (CEI)}

La variable centres de recherche juge le nombre d'institutions de recherche privées et publiques situées à l'intérieur ou autour du pôle. Une valeur 1 signifie l'absence ou une très courte présence de cette sorte d'agents.

\section{Variable 2: Offre de personnel technico-scientifique (OPCT)}

La deuxième variable concerne l'offre de personnel technico-scientifique dans la zone où se trouve le pôle. La valeur atteinte dans cette variable est directement corrélée avec la présence d'universités de taille et de prestige dans la ville. L'offre de ce type de personnel est souvent facilitée par l'existence d'un bassin d'entreprises innovatrices à la fine pointe de la technologie. 


\section{Variable 3: Essaimage de recherche (ER)}

Cette variable fait référence à la création de nouvelles entreprises par des chercheurs qui proviennent directement des centres de recherche ou des universités de la zone. La vision d'occasions d'affaires pour les chercheurs à partir des résultats obtenus par les sources de recherche où ils travaillent habituellement est la clé pour atteindre un bon taux d'essaimage de recherche.

\section{Variable 4: Collaboration université-entreprise (CUE)}

Cette variable est axée sur le degré réel de collaboration directe entre les entreprises locataires des pôles technologiques et les universités à proximité. Elle concerne plus particulièrement l'accès des entreprises du pôle à l'équipement matériel des départements universitaires, les connaissances et autres compétences intangibles détenues par les équipes de recherche.

\section{Variable 5: Spontanéité des relations (SO)}

Tandis que la variable précédente fait allusion au degré de collaboration ou d'usage des capacités universitaires par les entreprises, celle-ci fait le point sur la spontanéité de ces rapports, avec l'intention de découvrir si ces liens sont naturels et établis de façon autonome par les entreprises externes.

Pour mieux comprendre les résultats de l'étude empirique, le tableau $2 a$ sépare les pôles expérimentés ou matures, ceux ayant au moins 10 ans d'existence, et les pôles jeunes ou à l'état naissant.

On peut constater l'avantage logique des pôles plus matures sur les plus jeunes pour toutes les variables. La faiblesse des pôles récents est plus évidente pour les variables 1, 3 (à l'exception du remarquable résultat à Atlanpole Nantes) et 5, bien que les promoteurs de ces pôles s'attendent à un grand progrès au regard de ces variables dans les prochaines années.

La première colonne représente notre estimation chiffrée de la présence de centres de recherche dans le pôle ou en périphérie. Les résultats comparatifs sur ce sujet font apparaître la forte hétérogénéité des pôles visités en ce qui concerne leur importance scientifique et technique.

La Cité scientifique s'étendant sur un vaste territoire au cœur de la région Île-de-France est logiquement le milieu qui présente la plus forte concentration de centres de recherche et de formation supérieure, ainsi que la plus riche offre de personnel scientifique et technique qualifié. Cependant, ces résultats ne correspondent pas du tout à un niveau de synergies satisfaisant ni entre ces agents et ressources, ni avec le dense tissu d'entreprises de la zone. 
TABLEAU $2 a$

Portée des liens entreprise-recherche aux pôles technologiques Échelle : minimum 1 maximum 5

\begin{tabular}{lcccccc}
\hline & Var 1 & Var 2 & Var 3 & Var 4 & Var 5 & Total \\
\hline Pôles matures & 1 & 2 & 1 & 2 & 2 & 1,6 \\
Aston Science Park & 2 & 2 & 2 & 3 & 3 & 2,4 \\
Warwick Science Park & 3 & 3 & 2 & 4 & 3 & 3 \\
Surrey Research Park & 2 & 3 & 3 & 4 & 4 & 3,2 \\
Louvain-la-Neuve & 2 & 3 & 2 & 2 & 2 & 2,2 \\
Lyon Technopolis & 2 & 3 & 2 & 2 & 1 & 2 \\
Montpellier Technopole & 2 & 3 & 3 & 3 & 2 & 2,6 \\
Rennes Atalante & & & & & & \\
Cité Scientifique & 4 & 4 & 2 & 2 & 2 & 2,8 \\
$\quad$ Île-de-France Sud & $\mathbf{2 , 2}$ & $\mathbf{2 , 9}$ & $\mathbf{2 , 1}$ & $\mathbf{2 , 7}$ & $\mathbf{2 , 4}$ & $\mathbf{2 , 5}$ \\
\hline Pôles matures & & & & & & \\
\hline Pôles jeunes & 1 & 2 & 1 & 1 & 1 & 1,2 \\
Sheffield Science Park & 1 & 3 & 1 & 2 & 1 & 1,6 \\
Montpellier & 1 & 2 & 1 & 2 & 1 & 1,4 \\
$\quad$ Technopole Régionale & 2 & 3 & 4 & 2 & 1 & 2,4 \\
Savoie Technolac & 1 & 2 & 1 & 1 & 1 & 1,2 \\
Atlanpole Nantes & $\mathbf{1 , 2}$ & $\mathbf{2 , 4}$ & $\mathbf{1 , 6}$ & $\mathbf{1 , 6}$ & $\mathbf{1}$ & $\mathbf{1 , 6}$ \\
Angers Technopole & $\mathbf{1 , 9}$ & $\mathbf{2 , 7}$ & $\mathbf{1 , 9}$ & $\mathbf{2 , 3}$ & $\mathbf{1 , 8}$ & $\mathbf{2 , 1}$ \\
\hline Pôles jeunes & & & & & & \\
\hline Total & & & & & & \\
\hline
\end{tabular}

En qualité de pôle de recherche, Surrey Research Park apparaît en deuxième place en tant qu'espace ayant une forte concentration de centres de recherche.

La présence de centres de recherche dans le reste des pôles n'est pas spécialement imposante. Elle est même moindre que l'on aurait pu prévoir sur des territoires plutôt destinés à des activités de R-D.

L'essaimage de recherche enregistre des niveaux faibles dans presque tous les pôles visités, à l'exception d'Atlanpole Nantes, dont le succès relatif sur ce sujet est le fruit d'une stratégie axée sur la promotion de l'esprit d'entrepreneuriat parmi les chercheurs des écoles supérieures et des centres de recherche locaux.

Louvain-la-Neuve, avec un programme d'appui aux projets d'entreprises axés sur la R-D, tout comme Rennes Atalante, atteint aussi des résultats remarquables sur ce front. Les valeurs 1 et 2 atteintes par le reste des pôles témoignent de leur faiblesse au regard de cette troisième variable.

L'intensité des synergies et des échanges directs entre l'ensemble des entreprises du pôle et les agents de recherche locaux est aussi un indicateur fiable de la 
portée des liens entre les deux communautés. Grâce à leur parrainage universitaire, les meilleurs résultats sur cet aspect sont obtenus par Surrey Research Park et Louvain-la-Neuve, suivis par Rennes Atalante et Warwick Science Park.

De nouveau, les pôles les plus jeunes comme Angers Technopole et Sheffield Science Park affichent une plus faible trajectoire. Notons que le résultat d'autres pôles plus matures comme Aston Science Park, Montpellier Technopole, Lyon Technopolis et Atlanpole Nantes est aussi bas.

Toutes ces relations sont en général peu spontanées, ce qui rend évidente la nécessité de promouvoir des actions visant à stimuler la rencontre et l'échange d'idées entre les sources de recherche et le monde des entreprises.

À notre avis, ces liens ne sont véritablement spontanés que dans les pôles de Louvain-la-Neuve, Surrey Research Park et, dans une certaine mesure, Warwick Science Park.

Globalement, Louvain-la-Neuve, Surrey Research Park, Rennes Atalante, Warwick Science Park et Atlanpole Nantes sont les sites technologiques où la collaboration entre la recherche et les entreprises atteint des taux satisfaisants, sans toutefois mériter le qualificatif d'excellents. Tous représentent des projets expérimentés, lancés au début des années 1980, à l'exception d'Atlanpole Nantes, un pôle un peu plus récent.

Le cas de la Cité scientifique est caractérisé par des propriétés spéciales : sa bonne position relative provient d' une forte concentration d'institutions de recherche dans la région sur laquelle ce pôle est établi. Par contre, en ce qui concerne les rapports entre recherche et entreprises, la situation de la Cité n'est pas si positive, présentant un niveau semblable à la plupart des pôles de l'étude.

En ce qui concerne le reste des pôles, les liens de collaboration entre les entreprises et la recherche ne sont pas significativement plus forts que ceux établis avec des entreprises situées hors de ces espaces technologiques, du fait, dans la plupart des cas, de leur courte période d'existence.

La phase suivante de notre étude vise la réalisation d'une analyse factorielle effectuée sur le tableau $2 a$ pour définir les variables synthétiques qui expliquent les liens entre recherche et entreprises dans les pôles. Cette analyse fournit deux facteurs ou indicateurs synthétiques qui expliquent $82,4 \%$ de la variance totale du modèle.

Le facteur 1 garde une forte corrélation positive principalement avec l'offre de personnel scientifique et technique $(0,95)$ suivi par la présence des centres de recherche $(0,86)$. Pour ce motif, on l'appelle «Facteur d'intensité scientifiquetechnique». Ce facteur est donc de nature plutôt quantitative et matérielle. 
Analyse factorielle

\begin{tabular}{lcccccc}
\hline Variable & Communalité & $*$ & Facteur & $\begin{array}{c}\text { Valeur } \\
\text { propre }\end{array}$ & $\begin{array}{c}\% \\
\text { de variance }\end{array}$ & $\begin{array}{c}\% \\
\text { cumulatif }\end{array}$ \\
\hline CEI & 0,81848 & $*$ & 1 & 2,94931 & 59,0 & 59,0 \\
CUE & 0,92369 & $*$ & 2 & 1,17045 & 23,4 & 82,4 \\
ER & 0,92352 & $*$ & & & & \\
OPCT & 0,90166 & $*$ & & & & \\
SO & 0,55241 & $*$ & & & \\
VARIMAX & en trois itérations. & & & \\
Matrice des facteurs après rotation : & & & \\
& Facteur 1 & Facteur 2 & \\
CEI & 0,86107 & 0,27755 & & \\
CUE & 0,26765 & 0,92307 & & \\
ER & 0,14364 & 0,95021 & & \\
OPCT & 0,94911 & 0,02920 & \\
SO & 0,65064 & 0,35928 & & \\
\hline
\end{tabular}

Le facteur 2 est plutôt expliqué par l'essaimage de recherche $(0,95)$ et la portée de la collaboration entre l'université et l'entreprise $(0,92)$. On peut donc l'appeler «Facteur de relation recherche-entreprises du pôle », de nature tant quantitative que qualitative et immatérielle.

Le résultat de cette analyse factorielle autorise à penser que le véritable potentiel des liens entre les entreprises situées au sein d'un pôle technologique et les agents de recherche dépend fondamentalement de l'intensité technico-scientifique des travaux remplis par les institutions de recherche locales et la collaboration réelle entre la recherche et l'entreprise ainsi que la capacité des sources de recherche de créer de nouvelles entreprises innovatrices.

Par conséquent, ces facteurs devraient devenir les priorités des agents aménageurs des PT afin d'optimiser la présence et l'impact des centres de formation supérieure et de recherche auprès des pôles technologiques.

Nous poursuivons notre étude sur le tableau $2 a$, à partir duquel nous observons un contraste du degré de réalisation de ces deux facteurs ou des indicateurs synthétiques pour chaque pôle, comme le montre le tableau $2 b$ en opposant pôles matures et pôles jeunes.

De nouveau, et en utilisant le tableau $2 b$, le niveau d'explications du facteur 1 atteint par la Cité scientifique et l'intensité scientifique et technique du territoire d'accueil du pôle ressortent de façon notable. En effet, la région Île-de-France Sud, sur laquelle la Cité scientifique s'étend, absorbe presque la moitié de la capacité scientifique nationale. 
TABLEAU $2 b$

Facteurs synthèse des liens entreprise-recherche

\begin{tabular}{lrrr}
\hline & Facteur 1 & Facteur 2 & \multicolumn{1}{c}{ Total } \\
\hline Pôles matures & $-1,244$ & 0,156 & $-1,9675$ \\
Aston Science Park & $-0,740$ & 1,266 & 0,0987 \\
Warwick Science Park & 0,479 & 1,394 & 2,3306 \\
Surrey Research Park & 0,141 & 2,071 & 2,4825 \\
Louvain-la-Neuve & 0,355 & $-0,191$ & 0,4030 \\
Lyon Technopolis & 0,519 & $-0,756$ & 0,0734 \\
Montpellier Technopole & 0,558 & 0,406 & 1,3974 \\
Rennes Atalante & 2,130 & $-0,652$ & 2,9525 \\
Cité Scientifique Île-de-France Sud & $\mathbf{0 , 2 7 5}$ & $\mathbf{0 , 4 6 2}$ & $\mathbf{0 , 3 6 8 5}$ \\
\hline Total pôles matures & & & \\
\hline Pôles jeunes & $-0,237$ & $-0,764$ & $-1,2335$ \\
Montpellier Technopole Régionale & $-0,992$ & $-0,944$ & $-2,7247$ \\
Sheffield Science Park & $-1,081$ & $-0,407$ & $-2,2967$ \\
Savoie Technolac & 0,634 & 1,102 & 1,2066 \\
Atlanpole Nantes & $-0,992$ & $-0,944$ & $-2,7247$ \\
Angers Technopole & $\mathbf{0 , 4 4}$ & $-\mathbf{0 , 7 3 9}$ & $-\mathbf{0 , 5 8 9 3}$ \\
\hline Total pôles jeunes & $-\mathbf{0 , 0 8 2}$ & $-\mathbf{0 , 1 3 8}$ & $-\mathbf{0 , 1 1 0 4}$ \\
\hline Total pôles & &
\end{tabular}

Les résultats les plus faibles sont obtenus par les pôles les plus pauvres en centres de recherche, comme ceux d'Aston SP, Savoie Technolac, Angers Technopole et Sheffield SP. Le reste des pôles occupent une position intermédiaire.

On constate aussi une remarquable disparité entre les résultats du facteur 1 et ceux du facteur 2. Pour le deuxième facteur, rapport entre la recherche et les entreprises, les résultats les plus élevés sont en premier lieu pour Louvain-la-Neuve $(2,073)$, suivi de Surrey Research Park, de Warwick Science Park et d'Atlanpole Nantes. Tous les trois se maintiennent sensiblement au-dessus du reste des pôles. Le rôle joué par leur université respective a été décisif dans la configuration de ces trois espaces technopolitains, ce qui a permis aux rapports entre la recherche et les entreprises locataires de se développer d'une façon plus fluide. Le rôle joué par l'équipe animatrice des pôles à Nantes fut essentiel aux bons résultats dans l'essaimage de recherche.

Au contraire, la faible implication des universités locales à Sheffield Science Park et à Angers explique les moindres valeurs atteintes par ces pôles, ainsi que par les deux expériences technopolitaines de Montpellier et par la Cité scientifique, cette dernière souffrant du manque de liens entre les sources scientifiques et techniques et la direction de ce pôle. 
Il faut également noter la faiblesse des résultats à Sheffield Science Park, à Savoie Technolac et à Angers Technopole, reliée, dans ce dernier cas, au caractère naissant de leur université.

L'analyse par groupes de pôles montre une meilleure position pour les pôles matures, lesquels atteignent dans l'ensemble une légère ponctuation positive pour les deux facteurs. Toujours avec le groupe des pôles matures, on perçoit une dichotomie entre le comportement des pôles français, d'une part, et les pôles britanniques, de l'autre. Les premiers affichent une relativement bonne position pour le facteur 1 avec des ponctuations positives pour tous, tandis que leur position sur le facteur 2, le plus immatériel et qualitatif, est négative, sauf pour Rennes Atalante.

Par contre, les pôles britanniques matures suivent le comportement contraire avec des résultats négatifs sur le facteur matériel (facteur 1) et positifs sur le facteur 2, doté d'une composante plus relationnelle et immatérielle.

On ne perçoit pas une différence remarquable entre les pôles français matures et jeunes en ce qui concerne le facteur 2. Cela montre la faible progression des pôles de ce pays quant au contenu qualitatif des rapports représentés par ce facteur.

Finalement, la colonne qui résume les deux facteurs ${ }^{5}$ nous montre la Cité scientifique, Surrey Research Park et Louvain-la-Neuve en meilleures positions. On doit rappeler la forte concentration des centres de recherche dans la vaste zone comprise à l'intérieur de la ligne de démarcation de la Cité scientifique, alors que les deux autres pôles doivent leur succès à une remarquable collaboration entre université et entreprise.

Le niveau d'accomplissement de notre indicateur conjoint, à Atlanpole Nantes et à Rennes Atalante, pourrait aussi être qualifié de satisfaisant. Par contre, cet indicateur global devient négatif pour Sheffield Science Park et Aston Science Park parmi les pôles britanniques, et pour Angers Technopole, Savoie Technolac et Montpellier Régional, en France, tous ces espaces ne bénéficiant pas d'une véritable implication et participation active de la communauté locale de recherche.

Par groupe, les matures sont mieux placés malgré la diversité des résultats.

Les pôles les plus jeunes ou à l'état naissant offrent une position plus faible puisqu'ils n'ont pas eu le temps d'attirer de nombreux centres de recherche et la présence d'entreprises est encore récente, ce qui les empêche d'atteindre de bons résultats pour les deux facteurs.

5. On a utilisé une technique statistique généralement admise pour rassembler les deux groupes de facteurs. 


\section{Conclusion}

Pour conclure, il faut enfin remarquer la forte diversité entre les 13 expériences réelles de PT analysées en ce qui concerne le degré de participation des agents scientifico-technologiques du milieu local dans la promotion et le développement d'un espace technopolitain, et leur relation avec les entreprises implantées sur les pôles technologiques.

La méthodologie statistique employée nous a permis de tirer des conclusions et des leçons sur la trajectoire, le comportement et les perspectives sur ce sujet pour chacun des pôles visités, mesurés à travers des ponctuations qualitatives fondées sur nos études de terrain.

En plus, l'analyse factorielle nous a conduits à prodiguer des conseils et à émettre des recommandations sur les clés du succès des pôles technologiques. En particulier, l'intensité scientifique-technique (facteur 1) et l'essaimage et la relation réelle entre les agents de recherche et les entreprises du pôle (facteur 2 ) sont, d'après notre étude factorielle, les conditions les plus favorables pour avancer vers la participation optimale de la communauté scientifico-technologique locale dans tous les domaines de la vie d'un pôle technologique.

Notre analyse empirique des pôles visités nous révèle que le potentiel des universités et des centres de recherche en tant que sources de nouveaux projets d'entreprises innovatrices (essaimage) est réellement faible pour la plupart des zones examinées. Seuls Atlanpole Nantes et Rennes Atalante présentent des taux acceptables de création de nouvelles entreprises innovatrices directement créées dans l'environnement de recherches universitaires.

En général, les universités qui participent activement à la promotion d'un PT ne jouent qu'un rôle très marginal comme vivier de nouveaux projets d'entreprises susceptibles d'être accueillies dans les pôles technologiques. Souvent, le règlement universitaire dissuade les chercheurs potentiellement entrepreneurs, puisqu'il oblige tous ceux qui décident de participer activement à la mise en œuvre d'une entreprise à quitter l'université.

Tout semble indiquer que le maillage des liens efficaces et durables entre centres de formation supérieure et de recherche et les unités d'entreprises a besoin du développement d'habitudes de travail en commun et d'une confiance mutuelle acquise au fil des ans et sur une longue période de temps ${ }^{6}$.

En conséquence, l'établissement d'accords de collaboration entre les entreprises des pôles et les agents de recherche ne s'avère pas un exercice aussi spontané et aussi automatique que tendent à le croire les promoteurs des PT.

6. Constatation attribuable à Macdonald (1987). 
En revanche, les entreprises de haute technologie se sentent plus attirées par des flux d'information provenant d'autres entreprises que par les progrès scientifiques obtenus par la recherche des centres publics.

\section{Bibliographie}

Allaman (1992), «Un technopôle en forme : Nantes », Actualité Nantes, octobre.

BENKo, G. (1991), Géographie des technopôles, Paris, Masson.

BRUHAT, T. (1991), Vingt technopôles, un premier bilan, Paris, DATAR.

BRUHAT, T. (1993), «Innovations des technopoles dans les politiques technologiques régionales en France », Séminaire sur les principales expériences internationales de parcs scientifiques, Madrid, 22-23 mars.

BRUHAT, T., P. ESCORSA et al. (1995), «Étude comparative des parcs scientifiques en Europe: enjeux pour une politique communautaire d'innovation », Sprint Report, Luxembourg, Commission des communautés européennes.

Campagni, R. (1991), Innovation Networks : Spatial Perspectives, Londres, Belhaven Press.

CASTElls, M. et P. HALl (1994), Las tecnopolis del mundo. La formación de los complejos industriales del siglo XXI, Madrid, Alianza Editorial.

DECOSTER (1991), «Les phénomènes de technopolisation en Île-de-France Sud : les collaborations recherche-industrie », Paris, CNRS, juillet.

FRANCE TECHNOPOLES (1993), «Axes d'excellence des technopoles françaises de l'Arc Atlantique », Lyon.

GAFFARD, J.L. et al. (1987), La technopole comme espace créateur de technologie, Programme de Recherche technopoles et développement, coédité par le Centre d'économie régionale (CER), Université d'Aix-Marseille III et CNRS, Laboratoire d'analyses des transformations de l'appareil productif (LATAPSES), Université de Nice et CNRS, Laboratoire d'économie de sociologie du travail (LEST) et Fondation Sophia Antipolis, livre I, septembre.

GAMELla, M. (1988), « Parques tecnologicos e innovacion empresarial », Nuevas formas de promocion para la industria espanola, Fundesco, Madrid.

GiUNTA, F.J. (1993), «Relations between industry and the academic community », Séminaire sur les principales expériences internationales de parcs scientifiques, Madrid, 22-23 mars.

MACDONALD, S. (1987), «British science parks : reflections on the politics of high technology », $R \& D$ Management, vol. $17, \mathrm{n}^{\circ} 1$, p. 25-37.

Maillat, D., O. Crevoisier et B. LeCoQ (1991), «Réseaux d'innovation », Document interne, Groupe GREMI.

MARCH-CHORDÁ, I. (1995), « Technopolitan strategies : at the edge of an innovation-driven territorial approach », International Journal of Technology Management, vol. 10, $\mathrm{n}^{\text {os }}$ 7-8, p. 894-906. 
MARCH-CHORDÁ, I. (1996), «Towards the maturity stage : an insight into the performance of French technopoles », Technovation, vol. 16, n³ 3, p. 143-152.

MARCH-CHORDÁ, I. (1996), «Parques tecnológicos en el mundo: indicadores de resultados », Información Comercial Española. Revista de Economía, n ${ }^{\circ} 754$, p. 162-174.

PERRIN, J.C. (1991), «Réseaux d'innovation-milieux innovateurs, développement territorial ", Revue d'Économie Régionale et Urbaine, $\mathrm{n}^{\text {os }}$ 3-4.

QUÉviT, M. (1993), «Stratégies d'innovation et référents territoriaux », Revue d'Économie Industrielle, $\mathrm{n}^{\mathrm{0}} 64,2^{\mathrm{e}}$ trimestre, p. 38-57.

QUINTAS, P. et al. (1988), Science Parks and the Growth of High Technology Firms, Londres, Croom Helm.

QuinTAS, P. et al. (1991), High Tech Fantasies: Science Parks in Society, Science and Space, Londres, Routledge.

SAXENIAN, A. (1988), «The Cheshire cat's grin : innovation and regional development in England », Technology Review, février-mars.

VAN DIERDONCK, R. et al. (1991), «An assessment of science parks : towards a better understanding of their role in the diffusion of technological knowledge ", $R \& D$ Management, vol. 21, $\mathrm{n}^{\mathrm{0}} 2$, p. 35-46. 